\title{
Los lípidos en la nutrición del abulón azul, molusco con potencial de cultivo
}

\section{The lipids in the blue abalone's nutrition,}

Recibido: 3 de septiembre de 2013; aceptado: 19 de junio de 2014

\section{Eduardo Durazo Beltrán ${ }^{1}$}

Universidad Autónoma de Baja California

\section{Resumen}

Se evaluó el efecto del tipo y nivel de lípidos en el crecimiento y metabolismo de ácidos grasos en abulón azul. La primera parte del estudio se realizó en una granja comercial, donde se analizaron tres tratamientos de alimentación (alimento formulado, macroalga fresca y la combinación de ambos) sobre parámetros biológicos y composición de ácidos grasos de organismos juveniles en un periodo de 329 días. Los perfiles de ácidos grasos de los abulones sugieren su capacidad para sintetizar ácidos grasos poliinsaturados n-3 y n-6 de cadena larga a partir de precursores de cadena más corta. En el segundo experimento se evaluó el efecto de la fuente y nivel de ácidos grasos, con trece tratamientos de alimentación y el uso de cuatro aceites comestibles, sobre el crecimiento y composición de ácidos grasos en tejido muscular. No se determinó una relación significativa entre crecimiento y un determinado tipo o nivel de ácido graso, pero se establecieron requerimientos de ácidos grasos n-3 y n-6.

Palabras clave: lípidos, ácidos grasos, nutrición, abulón, cultivo.

\begin{abstract}
The effect of type and level of lipids in the growth and metabolism of fatty acids in blue abalone was studied. The first part of the study was conducted in a commercial farm, where three dietary treatments (formulated diet, seaweed and both) were evaluated on biological parameters and fatty acid composition in the tissue of juvenile abalone, over a 329-day period. The fatty acid profiles of the abalone suggest their ability to synthesize long chain polyunsaturated fatty acids $\mathrm{n}-3$ and n-6 from short-chained fatty acids. In the second experiment, the effect of lipid source and level, from four different edible oils, on growth and tissue fatty acid composition was evaluated. No significant differences in growth were found among abalone fed the different level or oil types but fatty acid requirements n-3 and n- 6 were determined.
\end{abstract}

Keywords: lipid, fatty acids, nutrition, abalone, culture.

\section{INTRODUCCIÓN}

Los lípidos son un grupo heterogéneo de sustancias orgánicas, presentes en organismos animales y vegetales, que comparten la propiedad de ser solubles en solventes orgánicos no polares como benceno, hexano y cloroformo, y ser insolubles en agua. Esta característica se deriva de la naturaleza hidrófoba de sus estructuras. En la nutrición acuícola animal, los lípidos tienen dos funciones principales; la primera, al ser la mayor forma de energía corporal almacenada a través de las reservas de triglicérido; la segunda, al formar una parte estructural importante de los componentes de la membrana celular a través de compuestos como los glicerofosfolípidos en complejos de lipoproteínas (Leaver et al., 2008). Los lípidos de la dieta pueden ser utilizados por los animales acuáticos para obtener energía, generada como ATP, a través del metabolismo oxidativo de ácidos grasos, y

\footnotetext{
${ }^{1}$ Doctor en Ciencias en Oceanografía Costera. Profesor de Carrera Titular C en la Facultad de Ciencias Marinas de la Universidad Autónoma de Baja California. Investigador Nacional Nivel 1. Sus líneas de investigación son: Nutrición y fisiología digestiva de organismos acuáticos. Bioquímica y metabolismo nutricional de organismos acuáticos en condiciones de cultivo. Correo electrónico: edurazo@uabc.edu.mx. Website: http://fcm.ens.uabc.mx/ edurazo/.
} 
como fuente de ácidos grasos esenciales. Lo anterior permite disponer de las proteínas para crecimiento. Los lípidos son una fuente compacta y eficiente de energía, lo cual le confiere ventajas a los animales acuáticos en épocas de escasez de alimento. En animales marinos, los lípidos muestran importancia en la flotabilidad, ésta se asocia con su baja densidad y al volumen que ocupan sin requerir osmoregulación, mantenimiento de estructuras corporales, como es el caso de la presencia de ácidos grasos de la serie omega-3, lo que permite tener fluidez de biomembranas a bajas temperaturas y en osmoregulación, ya que integran un espacio osmóticamente inactivo que no requiere un gasto de energía. Los lípidos son requeridos por los animales acuáticos principalmente como fuente de energía y en el aporte de moléculas polares necesarias en la formación de membranas celulares. Este carácter dual es reflejado en la compartamentalización de los lípidos corporales en tejido adiposo, integrado fundamentalmente por triacilglicéridos y lípidos de membranas celulares compuestos por lípidos polares, como los glicerofosfolípidos y colesterol. Los triacilglicéridos de animales acuáticos son similares a los de mamíferos terrestres, una característica general es que presentan ácidos grasos saturados o monoinsaturados en las posiciones sn1 y sn3 del glicerol y los ácidos grasos poliinsaturados (AGP) se concentran en la posición sn2 (Tocher, 2003). Los glicerofosfolípidos de biomembranas son particularmente ricos en AGP, los cuales se localizan en la posición 2 del esqueleto de gli- cerol, característica común en organismos eucariontes (Vance y Vance, 2008).

Los lípidos de la dieta deberán de suministrar los ácidos grasos esenciales n-3 y n-6 requeridos para propósitos estructurales o fisiológicos que el organismo no puede sintetizar. Ya que los organismos animales no tienen la capacidad de sintetizar de novo ácidos grasos con configuración n-3 o n-6 (figura 1), su contenido y balance en la dieta son de suma importancia para cubrir los requerimientos esenciales (Tocher, 2010).

La importancia nutricional de los ácidos grasos poliinsaturados se ha asociado al hecho que son factores dietéticos esenciales en todos los animales, tanto terrestres como acuáticos. La elongación de ácidos grasos ocurre por la adición de dos unidades de carbono al extremo carboxilo de la cadena. Esto se realiza por acción de dos sistemas diferentes, uno ocurre en el retículo endoplásmico, el cual requiere malonil-CoA; el otro, se realiza en la mitocondria con el aporte de acetil-CoA.

En la naturaleza, las rutas para insaturar ácidos grasos son universales y se presentan en animales superiores, bacterias, protozoarios, algas y levaduras. Sólo los organismos fotosintéticos pueden sintetizar ácidos grasos poliinsaturados de novo $\mathrm{n}-3$ y $\mathrm{n}-6$, los animales no presentan esta capacidad. Sin embargo, los peces de agua dulce pueden elongar e insaturar los ácidos grasos 18:2n-6 o 18:3n-3 provenientes de la dieta. Una deficiencia de AGP n-6 y n-3 se manifiesta en trastornos como interrupción del crecimiento y puede afectar la re-

Figura 1. Posición y tipo de instauración en un ácido graso de 18 carbonos

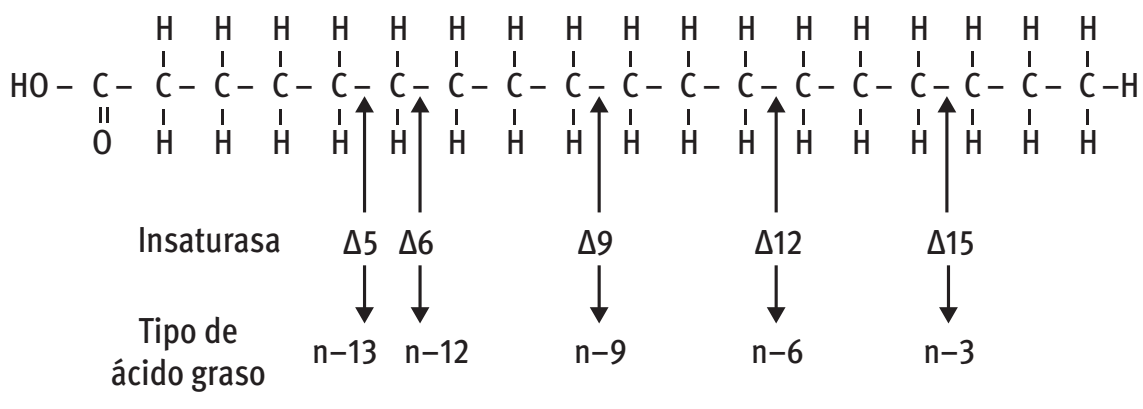

Fuente: elaboración propia. 
sistencia a enfermedades (Oliva-Teles, 2012). En plantas $\mathrm{y}$ animales terrestres las series de AGP $\mathrm{n}-6$ predominan sobre las n-3. En mamíferos terrestres se presentan altas concentraciones de AGP n-6 como el linoléico 18:2n-6 y el arquidónico 20:4-6 (Tacon et al, 2009). En tanto que en animales marinos, como peces, los AGP n-3 como el eicosapentaenoico 20:5n-3 y el docosahexaenoico 22:6n-3 presentan importancia para su desarrollo (Tocher, 2010). En los animales, los lípidos suministrados a través de la dieta muestran una mayor influencia sobre la composición de los lípidos corporales que el efecto del consumo de otro nutriente, como proteínas o carbohidratos. Esto se asocia a la baja especificidad de las interacciones enzima-sustrato en las rutas metabólicas a través de las cuales los lípidos son metabolizados (Sargent et al., 2002; Leaver et al., 2008). Como es el caso de insaturasas de ácidos grasos que muestran una baja especificidad de sustrato, donde la insaturasa $\Delta 6$ puede tener como sustratos a los ácidos grasos 18:1n-9, 18:2n-6, 18:3n-3, 24:5n-3 y 24:4n-6. Esta situación tiene como efecto que la conversión de AGP como 18:2n-6 a 18:3n-6 y 18:3n-3 a 18:4n-3, pueda competir con la conversión de ácidos grasos poliinsaturados de cadena larga (AGPCL) como 20:5n-3 a 22:6n-3 y 20:4n-6 a 22:5n-6, ya que la insaturasa $\Delta 6$ participa en dichas conversiones.
La competencia entre ácidos grasos de las familias n-3 y n-6 ocurre al nivel de insaturación y elongación de la cadena. A partir de estudios en mamíferos se reporta que la insaturasa $\Delta 6$ presenta una mayor afinidad por 18:3n-3 como sustrato con relación al 18:2n-6, por lo cual la abundancia de 18:3n-3 en la dieta puede interferir en la capacidad de formación de 20:4n-6 a partir de 18:2n-6.

En general la competencia entre 18:2n-6 y 18:3n-3 por la insaturasa $\Delta 6$ reduce la capacidad de formación y acumulación de ácidos grasos insaturados n-9. Sin embargo, en animales con deficiencia en ácidos grasos esenciales, la competencia no se presenta y ácidos grasos como 18:0 ó 18:1n-9 pueden ser utilizados como sustratos para reacciones de insaturación y elongación para generar compuestos indicadores de deficiencia como 18:2n-9 y 20:3n-9 (Miyazaki y Ntambi, 2008).

El aporte de ácidos grasos n-3 y n-6 y su balance en la dieta son de gran importancia para cubrir los requerimientos del organismo y favorecer su crecimiento. En el ambiente marino hay una diversidad de fuentes de AGPCL lo que ha generado que los organismos animales, de acuerdo al tipo de alimentación disponible en su entorno, hayan desarrollado diferentes capacidades para realizar reacciones de elongación e insaturación de AGP n-3 y n-6 y cubrir sus requerimientos (figura 2);

Figura 2. Elongación e insaturación de ácidos grasos en organismos animales

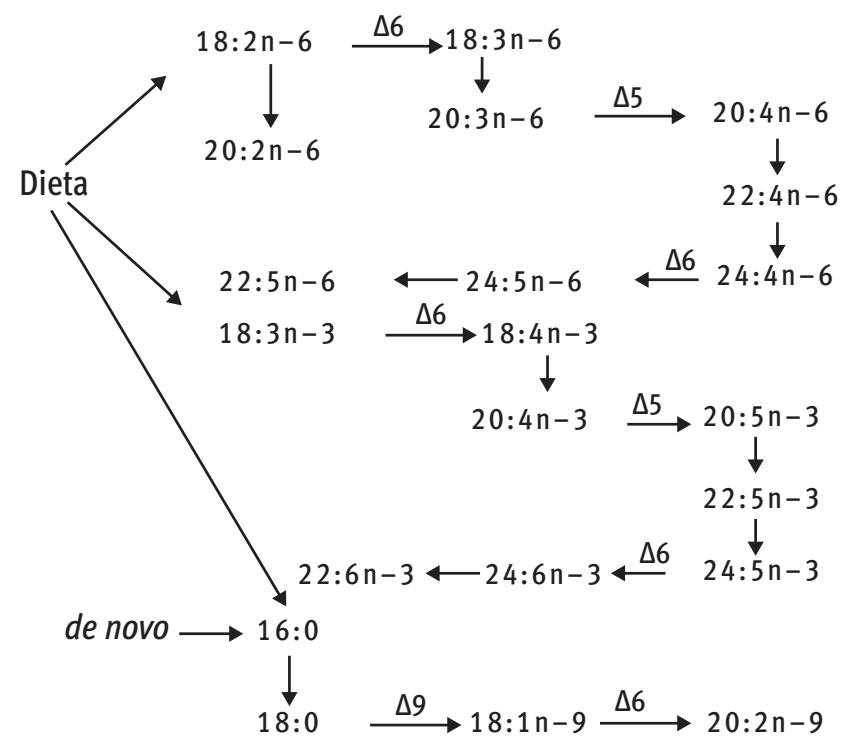

Fuente: Modificado de Miyazaki y Ntambi (2008).

(c) ENES Unidad León/UNAM 
en el caso de organismos marinos, como los peces, no presentan la capacidad enzimática para realizar dichas reacciones y cubrir sus necesidades metabólicas, por lo cual requieren el aporte de AGPCL en la dieta (Turchini et al., 2009).

Estudios de nutrición en condiciones de cultivo en el abulón (Haliotis spp.), molusco gasterópodo herbívoro marino (figura 3), han mostrado que el contenido y composición de los lípidos juegan un papel esencial en su crecimiento y composición tisular (Uki et al., 1986; Mai et al., 1995; Durazo et al., 2003; Green et al., 2011; BautistaTeruel et al., 2011; Hernández et al., 2013).

Figura 3. Juveniles de abulón azul (Haliotis fulgens)

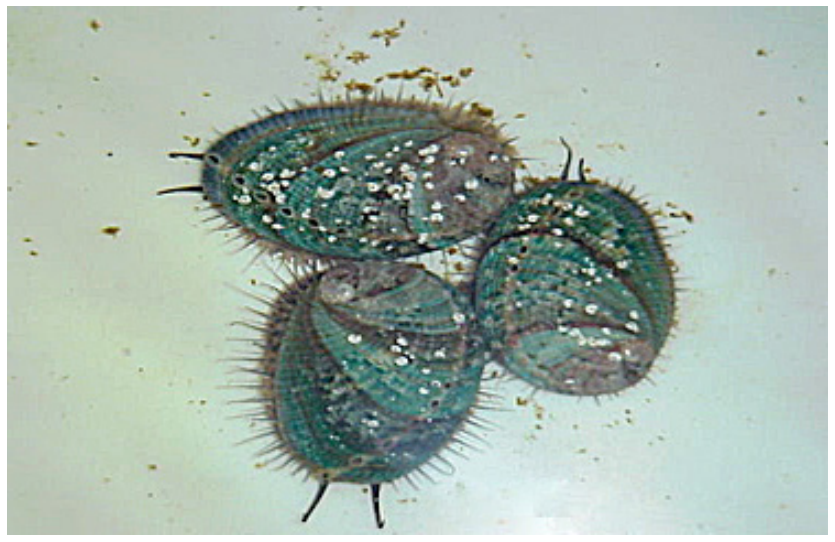

Con base en estudios con alimentos formulados se recomienda que la dieta para abulón contenga 3-5\% de lípidos, a efecto de favorecer tasas óptimas de crecimiento (Mai et al., 1995; Toledo-Agüero y Viana, 2009, BautistaTeruel et al., 2011). Sin embargo, se reporta que es posible obtener altas tasas de crecimiento con el uso de alimento formulado con $1.5 \%$ de lípidos de fuente marina (Fleming et al., 1996). En la nutrición del abulón, los lípidos de la dieta deben de cubrir principalmente los requerimientos de ácidos grasos n-3 y n-6 del organismo, el aporte energético no presenta preponderancia (Durazo y Viana, 2013). Con base en estudios en $H$. discus hannai, se estima que los requerimientos de AGPCL como 20:5n-3 y 22:6n-3 se cubren con un aporte en los lípidos de la dieta de $20 \%$ de $n-3$ y $2 \%$ de $n-6$.

Con el objetivo de aportar mayor conocimiento sobre el efecto y la importancia nutricional de ácidos grasos insaturados de cadena larga en el crecimiento, composición de ácidos grasos y metabolismo en el abulón azul (Haliotis fulgens) en condiciones de cultivo se realizó este estudio.

\section{DESARROLLO EXPERIMENTAL}

El estudio comprendió dos etapas: Experimento en condiciones de granja comercial y estudio a nivel laboratorio

\section{Experimento en granja comercial}

El estudio se realizó en una granja comercial de abulón (Ejido Eréndira, Baja California, México), donde juveniles de abulón azul, con un promedio de longitud concha de $5.9 \mathrm{~mm}$ y un peso de $24.2 \mathrm{mg}$, se sometieron durante 329 días a tres tratamientos de alimentación por duplicado, los cuales consistieron en el uso de alimento formulado experimental (tabla 1, figura 4), alimento natural (macroalga Macrocystis pyrifera, figura 5) y combinación de alimento formulado y alimento natural (figura 6).

Tabla 1. Contenido de ingredientes y composición proximal del alimento formulado experimental

\begin{tabular}{lc}
\hline \multicolumn{1}{c}{ Ingrediente } & $\mathrm{g} / 100 \mathrm{~g}$ \\
\hline Harina de pescado & 30.00 \\
Almidón de maíz & 14.66 \\
Harina de macroalga & 10.00 \\
Harina de maíz & 10.00 \\
Gelatina & 10.00 \\
Harina de soya & 8.00 \\
Celulosa & 5.00 \\
Almidón modificado & 5.00 \\
Mezcla de minerales & 4.00 \\
Mezcla de vitaminas & 1.50 \\
Ensilado de atún & 1.40 \\
Vitamina C & 0.20 \\
Cloruro de colina & 0.10 \\
Benzoato de sodio & 0.10 \\
Butilhidroxitolueno & 0.04 \\
\hline Composición proximal & $\%$ \\
\hline Carbohidratos & 47.3 \\
Proteína cruda & 29.0 \\
Cenizas & 11.9 \\
Lípidos totales & 5.9 \\
Humedad & 5.9 \\
\hline & \\
\hline
\end{tabular}

Fuente: elaboración propia.

El sistema de cultivo utilizado estuvo provisto de un flujo abierto y continuo de agua de $\operatorname{mar}\left(20 \mathrm{~L} \mathrm{~min}^{-1}\right)$, con aireación constante. La temperatura promedio del agua fue de $13.1^{\circ} \mathrm{C}$ en invierno y $21.1^{\circ} \mathrm{C}$ en verano. Los organismos se mantuvieron en seis tanques rectangulares 
de fibra de vidrio de 850 L con una densidad de 2000 abulones por tanque, con dos réplicas distribuidas al azar en el área de cultivo de la granja, suministrándose los tratamientos de alimentación ad libitum.

Figura 4. Alimento formulado experimental para abulón

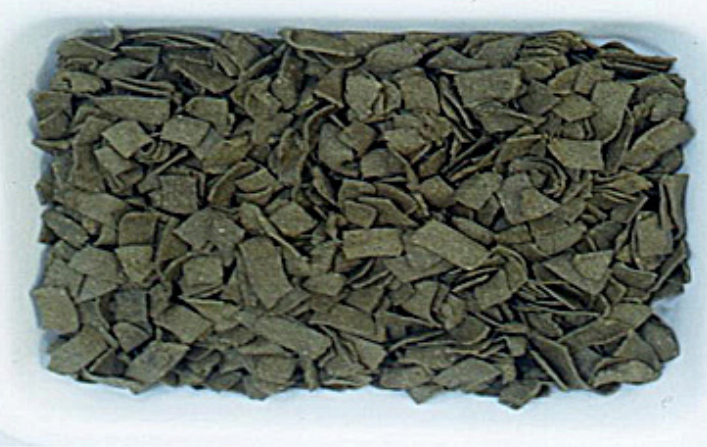

Figura 5. Macroalga Macrocystis pyrifera en el medio natural

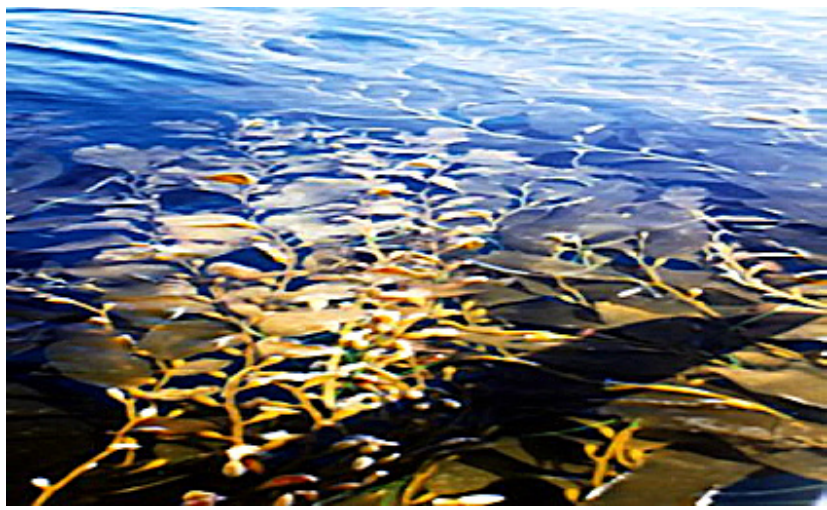

Figura 6. Abulón con alimento formulado y macroalga

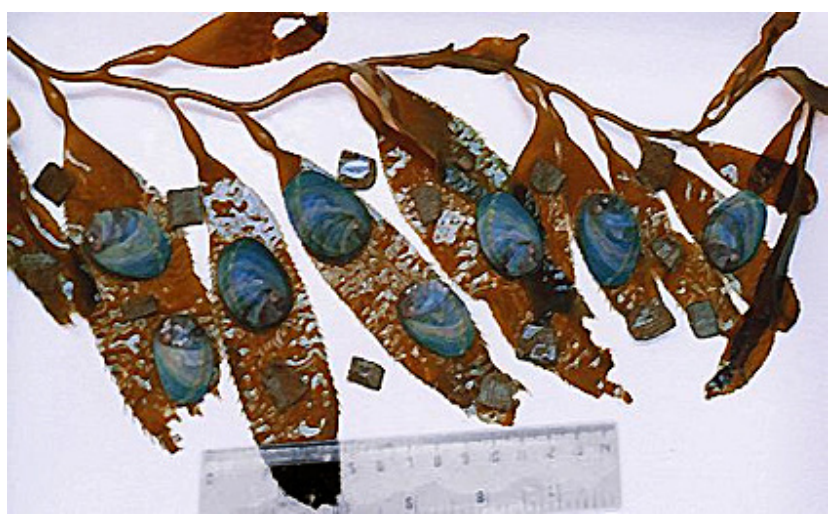

Se efectuaron al inicio, 141, 234 y 329 días del estudio mediciones de longitud de concha y peso total en 100 organismos tomados al azar de cada tanque, para estimar el crecimiento en longitud y en peso por tratamiento de alimentación. La supervivencia por tratamiento se efectuó por parte del personal de la granja, mediante el registro periódico de los organismos muertos. Al término del ensayo se tomaron 20 abulones por tanque para la extracción de lípidos totales (Folch et al., 1956) en tejido muscular por tratamiento y la posterior determinación del contenido de ácidos grasos. La determinación analítica de ácidos grasos se efectuó mediante cromatografía de gases capilar con detector de ionización de flama, los cuales se identificaron mediante estándares de ésteres metílicos de ácidos grasos bien caracterizados (Durazo y Viana, 2013). El análisis químico proximal de los alimentos se realizó por triplicado de acuerdo con métodos estándar (AOAC, 1995).

\section{Estudio en laboratorio}

La segunda parte del estudio se realizó en instalaciones de la Universidad Autónoma de Baja California campus Ensenada, donde se evaluó el efecto de alimentación balanceada con inclusión de diferentes niveles de lípidos y fuentes de ácidos grasos n-3 y n-6, a partir de aceites comestibles, sobre el crecimiento y la composición de ácidos grasos en tejido muscular. El experimento abarcó un ensayo de alimentación durante 75 días con abulones juveniles (19.7 mm de longitud media de concha y $910 \mathrm{mg}$ de peso promedio) obtenidos de una granja comercial, se utilizaron doce dietas experimentales (tabla 2) formuladas con cuatro aceites comestibles (oliva, maíz, linaza e hígado de bacalao) a tres niveles de concentración (1.5, 3.0 y $5.0 \%$ ) y tres concentraciones de tripalmitina (o, 2.0 у $3.5 \%)$. Las dietas fueron isoprotéicas (proteína cruda 38.4\%), isolipídicas (lípidos totales 5.1\%) e isocalóricas $(19.61 \mathrm{~kJ} / \mathrm{g})$. Se utilizó una dieta de referencia elaborada con ingredientes desgrasados con etanol, la cual presentó un bajo contenido lipídico (o.2\%). Los organismos se alimentaron con las dietas experimentales con una ración diaria equivalente a $2 \%$ de su peso corporal, se utilizó un sistema de cultivo con unidades de plástico de $3.8 \mathrm{~L}$ con un flujo $0.3 \mathrm{~L} / \mathrm{min}$ de agua de mar filtrada y aireada, a una temperatura promedio de $21.7^{\circ} \mathrm{C}$, con 10 organismos/unidad experimental. Los tratamientos de alimentación se evaluaron por triplicado.

Al inicio y al término del ensayo se determinó la longitud de concha y peso total de los abulones en cada unidad experimental, para calcular las tasas de creci- 
miento en longitud y en peso por tratamiento de alimentación. Para la determinación de la composición química proximal y el contenido de ácidos grasos en tejido muscular por tratamiento se tomaron 3 abulones por unidad experimental. El análisis químico proximal y la determinación de ácidos grasos de las muestras de alimentos y de tejido muscular de los abulones se realizaron como se describió previamente.

Tabla 2. Niveles de aceites y tripalmitina en dietas experimentales (g/100 g dieta)

\begin{tabular}{lccccc}
\hline Dieta & Oliva & Maíz & Linaza & $\begin{array}{c}\text { Hígado de } \\
\text { bacalao }\end{array}$ & Tripalmitina \\
\hline O5 & 5.0 & -- & -- & -- & -- \\
O3 & 3.0 & -- & -- & -- & 2.0 \\
O1.5 & 1.5 & -- & -- & -- & 3.5 \\
M5 & -- & 5.0 & -- & -- & -- \\
M3 & -- & 3.0 & -- & -- & 2.0 \\
M1.5 & -- & 1.5 & -- & -- & 3.5 \\
L5 & -- & -- & 5.0 & -- & -- \\
L3 & -- & -- & 3.0 & -- & 2.0 \\
L1.5 & -- & -- & 1.5 & -- & 3.5 \\
B5 & -- & -- & -- & 5.0 & -- \\
B3 & -- & -- & -- & 3.0 & 2.0 \\
B1.5 & -- & -- & -- & 1.5 & 3.5 \\
C & \multicolumn{5}{r}{ Sin adición de aceite o tripalmitina } \\
\hline
\end{tabular}

Fuente: elaboración propia.

Tabla 3. Composición proximal y energía bruta (E) de dietas experimentales (g/100 g dieta)

\begin{tabular}{lccccc}
\hline Dieta & $\begin{array}{c}\text { Proteína } \\
\text { cruda }\end{array}$ & $\begin{array}{c}\text { Lípidos } \\
\text { totales }\end{array}$ & Cenizas & $\begin{array}{c}\text { Carbo- } \\
\text { hidratos }\end{array}$ & E (kJ/g) \\
\hline O5 & 38.3 & 5.2 & 5.4 & 51.1 & 19.8 \\
O3 & 38.1 & 5.1 & 5.6 & 51.1 & 19.9 \\
O1.5 & 38.7 & 5.2 & 5.2 & 50.9 & 19.9 \\
M5 & 38.1 & 5.2 & 5.8 & 51.0 & 19.9 \\
M3 & 38.1 & 4.9 & 5.6 & 51.4 & 19.4 \\
M1.5 & 38.9 & 5.0 & 5.3 & 50.8 & 19.4 \\
L5 & 38.4 & 5.2 & 5.7 & 50.6 & 19.6 \\
L3 & 37.8 & 5.0 & 5.3 & 51.8 & 19.4 \\
L1.5 & 39.2 & 5.0 & 6.0 & 49.7 & 19.3 \\
B5 & 37.5 & 4.9 & 4.8 & 52.8 & 19.6 \\
B3 & 38.5 & 5.0 & 5.7 & 50.7 & 19.5 \\
B1.5 & 39.9 & 5.0 & 5.8 & 49.2 & 19.7 \\
C & 37.7 & 0.2 & 5.3 & 56.7 & 18.4 \\
\hline
\end{tabular}

Fuente: elaboración propia.

\section{RESULTADOS Y DISCUSIÓN}

\section{Experimento en granja comercial}

Bajo las condiciones de producción en la granja comercial, con un sistema de flujo de agua abierto, no fue posible determinar el consumo de alimento, ya que las unidades de cultivo presentaban un drenado hacia un colector común; además, la pérdida de materia seca del alimento formulado por el tiempo de permanencia en los tanques, por el fototactismo negativo del abulón, requiere ser estimada para precisar los valores del consumo alimenticio. Aun cuando no se evaluó el consumo de alimento, se estimó que el abulón se alimentó con las correspondientes dietas experimentales más que por pastoreo de microalgas que pueden estar presentes sobre las algas marinas y en las paredes de la unidad de cultivo; se observó que los abulones alimentados con la dieta combinada presentaron los mayores valores en longitud y en peso (5.8 mg/día) a lo largo del estudio (tabla 4). Así mismo, al término del ensayo, la mayor supervivencia (54\%) correspondió a la dieta combinada (figura 7 ).

Figura 7. Supervivencia (\%) de juveniles de abulón azul sometidos a tres tipos de alimentación durante 329 días

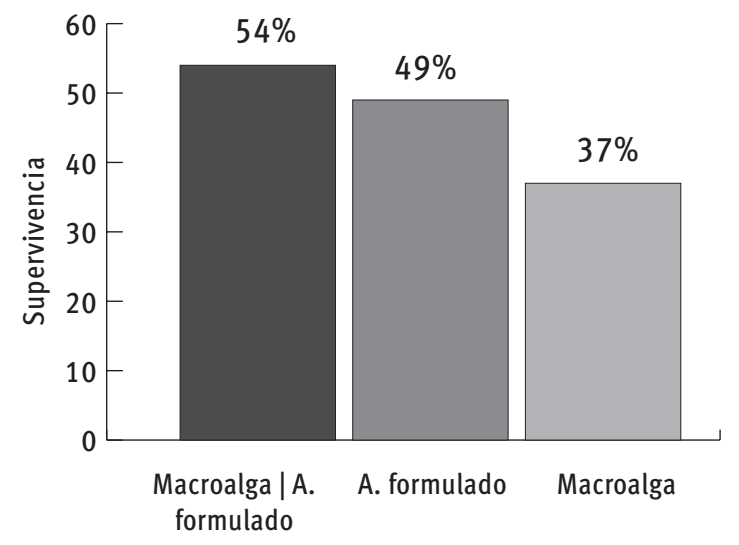

Fuente: elaboración propia.

La composición de los principales ácidos grasos de los alimentos y de la porción muscular de los abulones alimentados con los diferentes tratamientos se reportan en la tabla 5. Es de señalarse la ausencia de los AGPCL 22:4n-6 y 22:5n-3 en la macroalga, los cuales sí fueron detectados en los abulones alimentados con este tratamiento. En el alimento formulado no se detectó la presencia de 
Tabla 4. Peso (mg) y longitud de concha ( $\mathrm{mm})$ de juveniles de abulón azul (H. fulgens) sometidos a tres tratamientos de alimentación en condiciones de cultivo comercial

\begin{tabular}{|c|c|c|c|c|c|c|}
\hline \multirow{3}{*}{ Tratamiento } & \multicolumn{3}{|c|}{ Longitud (mm) } & \multicolumn{3}{|c|}{ Peso (mg) } \\
\hline & \multicolumn{3}{|c|}{ Días } & \multicolumn{3}{|c|}{ Días } \\
\hline & 141 & 234 & 329 & 141 & 234 & 329 \\
\hline Macroalga & $17.0 \pm 3.1$ & $18.4 \pm 3.2$ & $21.6 \pm 3.2$ & $457 \pm 4^{\mathrm{b}}$ & $805 \pm 16^{\mathrm{b}}$ & $1057 \pm 58^{\mathrm{b}}$ \\
\hline $\begin{array}{l}\text { Alimento } \\
\text { formulado }\end{array}$ & $15.3 \pm 2.9$ & $17.9 \pm 2.7$ & $20.7 \pm 3.0$ & $412 \pm 21^{b}$ & $695 \pm 18^{\mathrm{b}}$ & $985 \pm 36^{\mathrm{b}}$ \\
\hline $\begin{array}{l}\text { Macroalga }+ \\
\text { alimento } \\
\text { formulado }\end{array}$ & $18.9 \pm 3.1$ & $22.2 \pm 3.2$ & $25.2 \pm 3.3$ & $647 \pm 18^{\mathrm{a}}$ & $1242 \pm 57^{\mathrm{a}}$ & $1916 \pm 122^{a}$ \\
\hline
\end{tabular}

Promedio \pm desviación estándar. Diferentes letras por columna indican diferencias significativas $(\mathrm{P}<0.05)$

Fuente: elaboración propia.

22:4n-6 pero los abulones sometidos a este tratamiento de alimentación presentaron este ácido graso en su composición. Así mismo, en el tratamiento de alimentación combinada no se aportó el ácido graso 22:4n-6, pero se determinó su contenido en el perfil de ácidos grasos de los abulones sometidos a este tratamiento. Se determinó una correlación significativa entre los niveles de 22:4n-6 en músculo y 20:4n-6 en las dietas $\left(\mathrm{r}=0.99, \mathrm{P}=9.3 \times 10^{-5}\right)$, de la cual se puede especular que 22:4n-6 es producto de la elongación del ácido graso 20:4n-6. La acumulación de 22:5n-3 en tejido muscular con relación al contenido de este ácido graso en las dietas sugiere la capacidad de su biosíntesis a partir de precursores. Estudios en el abulón japonés (H. discus hannai) han establecido que esta especie es capaz de sintetizar 22:4n-6 y 22:5n-3 a partir de los precursores 18:2n-6 y 18:3n-3 (Uki et al., 1986; Floreto

Tabla 5. Contenido de ácidos grasos (\% del total de ácidos grasos) en alimento formulado, macroalga (M. pyrifera) y músculo de abulones alimentados con alimento formulado (AAF), macroalga (AMA) y alimentación combinada (AAF+MA) durante 329 días

\begin{tabular}{|c|c|c|c|c|c|}
\hline \multirow{2}{*}{ Ácido graso } & \multicolumn{2}{|c|}{ Alimento } & \multicolumn{3}{|c|}{ Músculo de abulón } \\
\hline & Formulado & Macroalga & $\mathrm{AAF}$ & AMA & $\mathrm{AMA}+\mathrm{AF}$ \\
\hline $14: 0$ & $5.6 \pm 0.1$ & $11.3 \pm 0.1$ & $3.0 \pm 0.0$ & $2.9 \pm 0.1$ & $2.9 \pm 0.1$ \\
\hline $16: 0$ & $24.3 \pm 0.1$ & $18.1 \pm 0.2$ & $17.3 \pm 0.2^{b}$ & $17.1 \pm 0.1^{\mathrm{b}}$ & $17.9 \pm 0.2^{\mathrm{a}}$ \\
\hline $18: 0$ & $6.6 \pm 0.1$ & $1.5 \pm 0.0$ & $6.4 \pm 0.1$ & $6.3 \pm 0.0$ & $6.4 \pm 0.1$ \\
\hline $18: 1 n-9$ & $18.7 \pm 0.1$ & $16.8 \pm 0.1$ & $5.3 \pm 0.1$ & $5.5 \pm 0.1$ & $5.5 \pm 0.1$ \\
\hline $18: 1 n-7$ & $3.6 \pm 0.0$ & nd & $6.3 \pm 0.1$ & $6.4 \pm 0.1$ & $6.3 \pm 0.3$ \\
\hline $18: 2 n-6$ & $21.4 \pm 0.1$ & $6.0 \pm 0.1$ & $6.3 \pm 0.2^{\mathrm{a}}$ & $3.9 \pm 0.0^{\mathrm{c}}$ & $4.4 \pm 0.0^{\mathrm{b}}$ \\
\hline $18: 3 n-3$ & $2.4 \pm 0.0$ & $3.3 \pm 0.1$ & $1.4 \pm 0.0^{\mathrm{a}}$ & $1.2 \pm 0.0^{\mathrm{b}}$ & $1.3 \pm 0.1^{\mathrm{a}}$ \\
\hline $18: 4 n-3$ & $0.2 \pm 0.0$ & $6.9 \pm 0.2$ & nd & nd & nd \\
\hline $20: 1 n-9$ & $1.7 \pm 0.1$ & $0.3 \pm 0.0$ & $0.6 \pm 0.0^{\mathrm{a}}$ & $0.4 \pm 0.0^{\mathrm{b}}$ & $0.6 \pm 0.0^{\mathrm{a}}$ \\
\hline $20: 4 n-6$ & $0.5 \pm 0.0$ & $18.2 \pm 0.2$ & $5.4 \pm 0.1^{\mathrm{c}}$ & $6.4 \pm 0.1^{\mathrm{b}}$ & $6.8 \pm 0.1^{\mathrm{a}}$ \\
\hline $20: 5 n-3$ & $0.6 \pm 0.0$ & $7.4 \pm 0.1$ & $5.0 \pm 0.1^{\mathrm{c}}$ & $7.1 \pm 0.0^{\mathrm{a}}$ & $6.0 \pm 0.1^{\mathrm{b}}$ \\
\hline $22: 4 n-6$ & nd & nd & $0.7 \pm 0.0^{\mathrm{b}}$ & $1.3 \pm 0.0^{\mathrm{a}}$ & $1.3 \pm 0.1^{\mathrm{a}}$ \\
\hline $22: 5 n-3$ & $0.2 \pm 0.0$ & nd & $6.9 \pm 0.1^{\mathrm{b}}$ & $8.7 \pm 0.1^{\mathrm{a}}$ & $8.6 \pm 0.1^{\mathrm{a}}$ \\
\hline $22: 6 n-3$ & $0.8 \pm 0.1$ & $0.2 \pm 0.0$ & $1.3 \pm 0.1^{\mathrm{a}}$ & $0.9 \pm 0.0^{\mathrm{b}}$ & $1.3 \pm 0.2^{\mathrm{a}}$ \\
\hline
\end{tabular}

Media \pm desviación estándar. nd : no detectado. 
et al., 1996). Además, se ha determinado qué especies como el abulón australiano (H. laevigata), el abulón de labio negro (H. rubra), el abulón tropical (H. assinina) y el abulón europeo (H. tuberculata) son capaces de producir ácidos grasos poliinsaturados de 20 carbonos a partir de precursores de 18 carbonos (Dunstan et al., 1996, BautistaTeruel et al., 2011; Hernández et al., 2013).

A través de un enfoque nutricional, los resultados del estudio a nivel de cultivo comercial de $H$. fulgens sugieren que la especie presenta la capacidad para la biosíntesis de ácidos grasos polinsaturados de cadena larga n-3 y n-6 a partir de ácidos grasos homólogos de cadena más corta.

\section{Estudio a nivel laboratorio}

El estudio desarrollado a nivel laboratorio estuvo enfocado a evaluar el efecto de dosis-respuesta de $H$. fulgens sometido a un gradiente en la dieta de 18:1n-9, 18:2n-6 y 18:3n-3 a partir de aceites vegetales, y de AGPCL n-3 derivados de aceite de pescado. Los resultados de crecimiento en este experimento no mostraron diferencias significativas con relación al tipo de aceite, sin embargo si se determinaron diferencias con relación al nivel de inclusión en el alimento. Los tratamientos con el nivel de $1.5 \%$ de aceite presentaron las mayores tasas de crecimiento, en tanto que con $5 \%$ se determinaron las menores (figura 8), esta diferencia no fue atribuible al consumo de alimento ya que las tasas de consumo entre los tratamientos fueron similares. Este efecto inhibitorio del crecimiento se puede atribuir a un exceso de ácidos grasos n-3 y/o n-6 con relación a los requerimientos del organismo, situación que ha sido reportada en especies acuáticas como peces (Takeuchi, 1997) y camarón (González-Félix et al., 2002).

Figura 8. Efecto del \% de inclusión de aceite en el alimento en el crecimiento en peso ( $\mathrm{mg} /$ día) de juveniles de $\boldsymbol{H}$. fulgens, al término de 75 días de alimentación

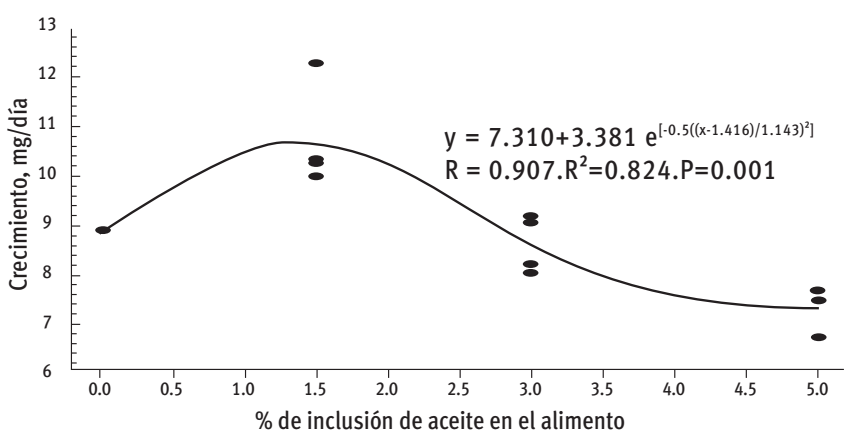

Fuente: elaboración propia.
Con base en un análisis de regresión se determinó que todos los tratamientos con un nivel de aceite de $1.5 \%$ se asociaron con los mayores crecimientos en peso y en longitud. Estos resultados contrastan con la recomendación de la inclusión de un nivel 3-5\% de lípidos en alimentos formulados para abulón para favorecer un máximo crecimiento (Thongrod et al., 2003; BautistaTeruel et al., 2011), en la cual es posible que se tenga un exceso de ácidos grasos n-3 y/o n-6. En este estudio no se pudo establecer una relación significativa entre crecimiento con un determinado tipo o nivel de ácido graso o con una relación n-3/n-6 suministrados en las dietas. Sin embargo, resultados de crecimiento mostraron que los abulones alimentados durante 75 días con la dieta de referencia ( $0.2 \%$ de lípidos totales) presentaron una ganancia en peso de $120 \%$ con relación al inicio del experimento, con un consumo de 2, 0.2, 0.2 y $0.2 \mu \mathrm{g} / \mathrm{g}$ de peso vivo/día de 18:2n-6, 18:3n-3, 20:4n-6 y 22:6n-3, respectivamente. En tanto, los organismos alimentados con la dieta con 1.5\% de aceite de maíz (5\% de lípidos totales) mostraron una ganancia en peso de $170 \%$, con un consumo de 9.1, 2, 1 y $0.1 \mu \mathrm{g} / \mathrm{g}$ de peso vivo/día de 18:2n-6, 18:3n-3, 20:4n-6 y 22:6n-3, respectivamente. Lo anterior indica que el abulón azul presenta un nivel bajo en su requerimiento de ácidos grasos n-3 y n-6. Mediante un análisis de regresión entre los niveles de 18:2n-6, 18:3n-3, 20:4n-6, 22:4n-6, 22:5n-3 y 22:6n-3 encontrados en músculo de los abulones (tabla 6) y los contenidos de 18:2n-6 y 18:3n-3 en los tratamientos de alimentación se determinaron regresiones significativas $(\mathrm{P}<0.05)$ entre estos (tabla 7).

A partir de estos resultados se puede sugerir que $H$. fulgens muestra la capacidad para elongar e insaturar 18:2n-6 hasta 22:4n-6, aun cuando no se detectó 18:3n-6 en músculo se estima que dicha bioconversión puede ocurrir mediante la acción de la insaturasa $\Delta 5$ por un mecanismo similar al reportado por Li et al. (2013). El efecto de dosis-respuesta del contenido de 18:3n-3 en el alimento con relación al contenido de 18:3n-3 en músculo fue manifiesto, obteniéndose una regresión significativa de $2^{\circ}$ orden con altos coeficientes de correlación y determinación ( $\left.R=0.979, R^{2}=0.959\right)$. Sin embargo, en el caso de 20:5n-3 y 22:5n-3 en músculo no se encontró correlación o regresión significativas con el 18:3n-3 presente en el alimento o entre 18:3n-3 en músculo con AGPCL n-3 presentes en músculo. Sólo se determinaron regresiones 
Tabla 6. Contenido de ácidos grasos (\% del total de ácidos grasos) n-6 y n-3 en las dietas experimentales elaboradas con cuatro aceites comestibles y en tejido muscular de abulones al término de 75 días de alimentación

\begin{tabular}{|c|c|c|c|c|c|c|c|c|c|}
\hline \multirow{3}{*}{ Dieta } & \multicolumn{9}{|c|}{ Ácido graso } \\
\hline & \multicolumn{2}{|c|}{ Alimento } & \multicolumn{7}{|c|}{ Músculo } \\
\hline & $18: 2 n-6$ & $18: 3 n-3$ & $18: 2 n-6$ & $18: 3 n-3$ & $20: 4 n-6$ & $20: 5 n-3$ & $22: 4 n-6$ & $22: 5 n-3$ & $22: 6 n-3$ \\
\hline O5 & 5.3 & 0.5 & 5.3 & 0.9 & 5.0 & 5.8 & 0.6 & 6.0 & 1.4 \\
\hline $\mathrm{O} 3$ & 3.3 & 0.3 & 4.4 & 1.0 & 5.1 & 5.4 & 0.6 & 5.4 & 0.8 \\
\hline 01.5 & 2.0 & 0.2 & 3.1 & 1.0 & 4.6 & 5.2 & 0.9 & 5.2 & 0.9 \\
\hline M5 & 46.4 & 0.7 & 9.1 & 0.7 & 8.0 & 4.0 & 1.6 & 5.8 & 0.9 \\
\hline M3 & 27.2 & 0.5 & 7.0 & 0.6 & 8.2 & 3.9 & 2.3 & 5.2 & 0.8 \\
\hline M1.5 & 14.2 & 0.3 & 5.6 & 0.9 & 7.5 & 5.3 & 1.9 & 6.1 & 0.8 \\
\hline L5 & 13.2 & 39.3 & 2.5 & 4.9 & 5.4 & 6.5 & 0.9 & 6.8 & 0.7 \\
\hline L3 & 8.0 & 22.9 & 2.4 & 4.4 & 6.8 & 9.0 & 1.3 & 8.7 & 0.9 \\
\hline L1.5 & 4.3 & 11.3 & 2.0 & 3.3 & 5.1 & 8.7 & 0.9 & 8.0 & 0.8 \\
\hline B5 & 3.4 & 0.9 & 1.4 & 0.3 & 3.4 & 10.7 & 0.4 & 8.3 & 2.8 \\
\hline B3 & 2.2 & 0.6 & 1.9 & 0.5 & 3.9 & 9.5 & 0.6 & 8.3 & 2.7 \\
\hline B1.5 & 1.5 & 0.3 & 1.9 & 0.8 & 3.9 & 9.8 & 0.5 & 8.0 & 2.4 \\
\hline
\end{tabular}

Fuente: elaboración propia.

Tabla 7. Regresiones entre contenidos de ácidos grasos insaturados en el alimento y en el músculo de abulón

\begin{tabular}{cccccc}
\hline Variable independiente & Variable dependiente & Ecuación & $\mathrm{R}$ & $\mathrm{R}^{2}$ & $\mathrm{P}$ \\
\hline 18:2n-6 en alimento & $18: 2 \mathrm{n}-6$ en músculo & $\mathrm{y}=1.415+0.177 \mathrm{x}$ & 0.855 & 0.731 & 0.002 \\
18:2n-6 en alimento & 20:4n-6 en músculo & $\mathrm{y}=2.34+0.30 \mathrm{x}+0.008 \mathrm{x}^{2}$ & 0.878 & 0.771 & 0.001 \\
18:2n-6 en músculo & $20: 4 \mathrm{n}-6$ en músculo & $\mathrm{y}=2.119+0.518 \mathrm{x}$ & 0.732 & 0.535 & 0.004 \\
18:2n-6 en músculo & $22: 4 \mathrm{n}-6$ en músculo & $\mathrm{y}=0.22+0.164 \mathrm{x}$ & 0.668 & 0.446 & 0.013 \\
20:4n-6 en músculo & $22: 4 \mathrm{n}-6$ en músculo & $\mathrm{y}=-0.458+0.319 \mathrm{x}$ & 0.919 & 0.845 & $<0.0001$ \\
18:3n-3 en alimento & $18: 3 \mathrm{n}-3$ en músculo & $\mathrm{y}=0.435+0.329-0.01 \mathrm{x}^{2}$ & 0.979 & 0.959 & $<0.0001$ \\
20:5n-3 en músculo & $22: 5 \mathrm{n}-3$ en músculo & $\mathrm{y}=0.557+0.604 \mathrm{x}$ & 0.941 & 0.885 & $<0.0001$ \\
22:5n-3 en músculo & $22: 6 \mathrm{n}-3$ en músculo & $\mathrm{y}=-0.341+0.300 \mathrm{x}$ & 0.598 & 0.357 & 0.031 \\
\hline
\end{tabular}

Fuente: elaboración propia.

significativas entre los contenidos de 20:5n-3, 22:5n-3 y 22:6n-3 de músculo.

Hay que señalar que no se detectaron los intermediarios 18:4n-3 y 20:4n-3, los cuales se relacionan con la síntesis de AGPCL n-3 a partir de 18:3n-3. Esto no se asociaría con una inhibición de la insaturasa $\Delta 6$, ya que esta enzima muestra una mayor afinidad de sustrato por AGP n-3 con relación a los ácidos grasos n-9 o n-6 (Zheng et al., 2004). Si se considera que el mecanismo de elongación presenta una baja especificidad de sustrato (Miyazaki y Ntambi, 2008) y se detectó elongación en la síntesis de AGPCL n-6, se estima que la ausencia de 18:4n3 y 20:4n-3 en la composición del músculo del abulón podría estar relacionada con su rápida conversión a un homólogo superior.
A partir del estudio de requerimientos de ácidos grasos poliinsaturados en abulón azul a través de ensayos de alimentación se estableció lo siguiente:

a) $H$. fulgens alimentado con dietas balanceadas con $1.5 \%$ de aceite de oliva, maíz, linaza o hígado de bacalao presenta tasas de crecimiento mayores con relación a dietas con un contenido de $5 \%$;

b) juveniles de abulón azul no muestran inhibición en su crecimiento con el consumo de 2, 0.2, 0.2 y 0.2 $\mu \mathrm{g} / \mathrm{g}$ de peso vivo/día de los ácidos grasos 18:2n-6, 18:3n-3, 20:4n-6 y 22:6n-3, respectivamente;

c) $H$. fulgens presenta la capacidad para sintetizar AGPCL n-3 y n-6 a partir de ácidos grasos precursores de series homólogas de menor longitud de cadena y menor grado de insaturación. 
El aporte del trabajo mediante un enfoque nutricional se desarrolló para contar con un mayor conocimiento sobre el efecto de los lípidos y ácidos grasos de la dieta en el crecimiento, depositación y metabolismo de una especie con potencial para su cultivo comercial como es el abulón azul. A partir de este conocimiento se podrán desarrollar alimentos balanceados que permitan eficientizar el uso de fuentes lipídicas que favorezcan una mejor nutrición de $H$. fulgens en condiciones de cultivo y en las diferentes etapas de crecimiento del organismo. El desarrollo de posteriores estudios sobre metabolismo digestivo de lípidos a través de la caracterización de lipasas, tasas de depositación de lípidos en tejidos, evaluación de la digestibilidad de lípidos, efecto de la actividad microbiana en sistema digestivo y caracterización de la expresión génica de insaturasas en permitirán un mayor conocimiento sobre la especie bajo estudio.

\section{REFERENCIAS}

AOAC (1995). Association of Official Analytical Chemists. Official Methods of Analysis of AOAC International. Vol. 1. Arlington: AOAC International.

Bautista-Teruel, M., Koshio, S., y Ishikawa, M. (2011). Diet development and evaluation for juvenile abalone, Haliotis asinina Linne: Lipid and essential fatty acid levels. Aquaculture, 312, 172-179.

Durazo, E., D’Abramo, L., Toro-Vásquez, J., VasquezPeláez, C., y Viana, M. (2003). Effects of triacylglycerols in formulated diets on growth and fatty acid composition in tissue of green abalone. Aquaculture, 224, (1-4). 257-270.

Durazo, E., y Viana, M. (2013). Fatty acid profile of cultured green abalone (Haliotis fulgens) exposed to lipid restriction and long-term starvation. Ciencias Marinas, 39, (4). 363-370.

Dunstan, G., Baillie, H., Barrett, S., y Volkman, J. (1996). Effect of diet on the lipid composition of wild and cultured abalone. Aquaculture, 140, 115-127.

Fleming, A., Van Barneveld, R., y Hone, P. (1996). The development of artificial diets for abalone: A review and future directions. Aquaculture, 140, (1). 5-63.

Floreto, E., Teshima, S., y Koshio, S. (1996). The effects of seaweed diets on the lipid and fatty acid of the
Japanese disc abalone Haliotis discus hannai. Fisheries Science, 62, (4). 582-588.

Folch, J, Lees, M., y Stanley, S. (1957). A simple method for the isolation and purification of total lipids from animal tissues. The Journal of Biological Chemistry, 226: 497-509.

González-Félix, M., Gatlin III, D., Lawrence, A., y Perez-Velazquez, M. (2002). Effect of various dietary lipid levels on quantitative essential fatty acid requirements of juvenile Pacific white shrimp Litopeneaus vannamei. Journal of the World Aquaculture Society, 33, 330-340.

Green, A., Jones, C., y Britz, P. (2011). Effect of dietary lipid level on growth and feed utilization in cultured South African abalone Haliotis midae L. fed diets with a constant protein-to-energy ratio. Aquaculture Research, 42, (10). 1501-1508.

Hernández, J., Matus-de la Parra, A., Lastra, M., y Viana, M. (2013). Effect of lipid composition of diets and environmental temperature on the performance and fatty acid composition of juvenile European abalone (Haliotis tuberculata L. 1758). Aquaculture, 412-413, 34-40.

Leaver, M., Bautista, J., Bjornsson, B., Jonsson, E., Krey, G., Tocher, D. y Torstensen, B. (2008). Towards fish lipid nutrigenomics. Reviews in Fisheries Science, 16, 73-94.

Li, M., Mai, K., He, G., Ai, Q., Zhang, W., Xua, W., Wang, J., Liufu, Z., Zhang, Y., y Zhou, H. (2013). Characterization of two $\Delta 5$ fatty acyl desaturases in abalone (Haliotis discus hannai Ino). Aquaculture, 416-417, 48-56.

Mai, K., Mercer, J. y Donlon (1995). Comparative studies on the nutrition of two species of abalone, Haliotis tuberculata L. and Haliotis discus hannai Ino. III. Response of abalone to various levels of dietary lipid. Aquaculture, 134, (1). 65-80.

Miyazaki, M., y Ntambi, J. (2008). Fatty acid desaturation and chain elongation in mammals, en Vance, D., Vance, J. (Eds.). Biochemistry of Lipids, Lipoproteins and Membranes. Amsterdam: Elsevier.

Oliva-Teles, A. (2012). Nutrition and health of aquaculture fish. Journal of Fish Diseases, 35, 83-108. Sargent, J., Tocher, D. y Bell, J. (2002). The lipids, en Halver, J. y Hardy, R. (Eds.). Fish Nutrition (pp. 181257). Estados Unidos: Elsevier. 
Tacon, A., Metian y M., Hassan, M. (2009). Feed ingredients and fertilizers for farmed aquatic animals: sources and composition. FAO Fisheries and Aquaculture Technical Paper, 540.

Takeuchi, T. (1997). Essential fatty acid requirements of aquatic animals with emphasis on fish larvae and fingerlings. Reviews in Fisheries Science, 5, (1). 1-25.

Tocher, D. (2003). Metabolism and functions of lipids and fatty acids in teleost fish. Reviews in Fisheries Science, 11, (2). 107-184.

(2010). Fatty acid requirements in ontogeny of marine and freshwater fish. Aquaculture Research, 41, 717-732.

Toledo-Agüero, P., y Viana, M. (2009). Composición de ácidos grasos en juveniles de abulón Haliotis tuberculata coccinea alimentados con dietas formuladas con diferentes contenidos de HUFA n3. Ciencias Marinas, 35, (1). 101-112.

Thongrod, S., Tamtin, M., Chairat, C., y Boonyaratpalin, M. (2003). Lipid to carbohydrate ratio in donkey's ear abalone (Haliotis asinina, Linne) diets. Aquaculture, 225, 165-174.

Turchini, G., Torstensen, B., y Ng, W. (2009). Fish oil replacement in finfish nutrition. Reviews in Aquaculture, 1, 10-57

Uki, N., Sugiura, M., y Watanabe, T. (1986). Requirement of essential fatty acids in the abalone Haliotis discus hannai. Bulletin of the Japanese Society of Scientific Fisheries, 52, (6). 1013-1023.

Vance, D., y Vance, J. (2008). Phospholipid biosynthesis in eukaryotes, en Vance, D., Vance, J. (Eds.). Biochemistry of Lipids, Lipoproteins and Membranes (c. 8.). 5 a ed., Oxford: Elsevier.

Zheng, X., Seiliez, I., Hastings, N., Tocher, D., Panserat, S., Dickson, C., Bergot, P., y Teale, A. (2004). Characterization and comparison of fatty acyl D6 desaturase cDNAs from freshwater and marine teleost fish species. Comparative Biochemistry and Physiology, Part B 139, 269-279. 